\title{
Turbulent air flow field in slot-die melt blowing for manufacturing microfibrous nonwoven materials
}

\author{
Sheng Xie ${ }^{1, *}\left(\mathbb{C}\right.$, Wanli $\operatorname{Han}^{1} \oplus$, Guojun Jiang ${ }^{2} \oplus$, and Chao Chen ${ }^{1} \oplus$ \\ ${ }^{1}$ College of Material and Textile Engineering, Jiaxing University, Jiaxing 314001, People's Republic of China \\ ${ }^{2}$ Zhijiang College of Zhejiang University of Technology, Hangzhou 310024, People's Republic of China
}

Received: 13 November 2017

Accepted: 6 January 2018

Published online:

16 January 2018

(C) The Author(s) 2018. This article is an open access publication

\begin{abstract}
Melt blowing is an industrial approach for producing microfibrous nonwoven materials utilizing high-speed air to attenuate polymer melt. The melt-blowing air flow field which is widely believed to be turbulence determines the process of fiber formation. In this study, the turbulent air flow field in slot-die melt blowing was experimental measured by hot-wire anemometer. The fluctuations of air velocity and temperature, the mean velocity and mean temperature were measured and analyzed; moreover, the relationship between turbulent air flow field and fiber formation in melt blowing was discussed and predicted. In the last part of this paper, the coupling effect of air temperature and velocity was studied tentatively, results showed that air temperature not only had an enhanced effect on velocity, but contributed to the fluctuation of velocity. This work shows that the fluctuating characteristics of air velocity and temperature have dominant effect on fiber motion and the evenness of fiber diameter.
\end{abstract}

\section{Introduction}

Melt blowing is one of industrial approaches for manufacturing nonwoven materials. Because of the fiber diameter produced by melt blowing is commonly in the range from about $1 \mu \mathrm{m}$ to several micrometers, the melt-blown nonwoven materials have found a variety of advanced applications in areas of filtration, life science, medicine and industry [1, 2].

During melt-blowing process, the microfibers are manufactured by extruding a polymer melt through an orifice (a component of die), and attenuating the extrudate with a jet of high velocity hot air (see
Fig. 1). Obviously, the air flow field plays a crucial role in fiber formation during melt blowing. Until now, the air flow fields in melt blowing have been researched mostly by methods of computational fluid dynamics (CFD) numerical simulation and experimental measurement. Shambaugh and his co-workers [3-7] have systematically numerical simulated the air flow fields in different kinds of die melt blowing, including slot die, annular die and swirl die. Their numerical simulations obtained the profiles of the whole air flow fields including the profiles of air velocity and temperature. Not only this, they have also experimental measured the air velocity and temperature below the die, using a Pitot tube and a

Address correspondence to E-mail: shengxie16@163.com 
Figure 1 a Schematic of the process of slot-die melt blowing [21], $\mathbf{b}$ the structure of single-orifice slot die and, c the real single-orifice slot die used in this work. The configuration of the air channel is similar to the letter of ' $\mathrm{V}$ ', and this kind of die is also called V-slot die.

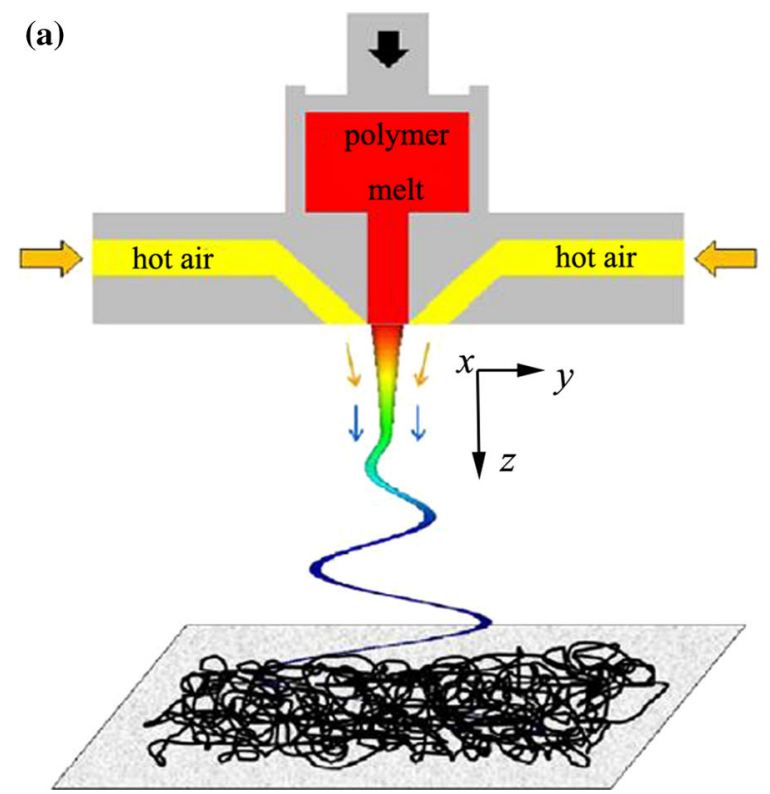

(b)

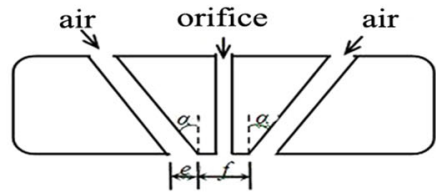

(c)

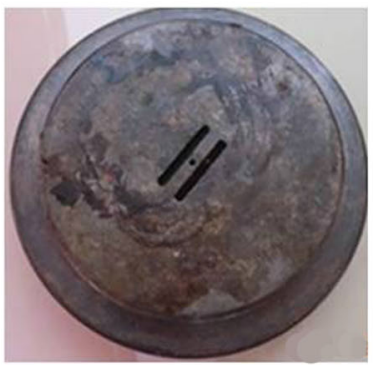

thermocouples (or infrared thermometer), respectively [8-11]. However, for the limitation of Pitot tube, air velocities they measured were far below the industrial level, about velocity of sound [12]. Later, more advanced equipments with higher accuracy, such as hot-wire anemometer and laser Doppler velocimeter were applied to measuring the air flow fields [13-16], the maximum velocity they measured increased to be about $150 \mathrm{~m} \mathrm{~s}^{-1}$. It noted that the air flow fields all they have measured were described by average values, namely, the turbulent characteristic of air flow field was not provided.

In commercial slot-die melt blowing, after the two streams of high velocity hot air are extruded out from the air channels of the die, they undergo the processes of converging, merging and combining (as shown in Fig. 2a) [17], illustrating that the air flow field in melt blowing has obvious characteristic of turbulence. Research group of Kumar and Bates [18-22] has done some theoretical and experimental work on the fiber formation, onset of fiber whipping and fiber breakup as well as utilizing an innovative Laval nozzle in melt blowing, their work had a significant contribution to the research on melt blowing, and they also mentioned that, in melt blowing, whipping-like motion of fiber was due to turbulent air [20]. Furthermore, the fiber motion in melt blowing can indirectly reflects the turbulent characteristic of air flow field. Vibration of fiber was firstly recorded by Shambaugh and his co-workers [23], at that time, a bundle of fibers appeared to be splaying rather than single fiber were captured. Bresee et al. [24-26] also devoted some experimental measurements to fiber motion using the equipment of highspeed photography. Xie and Zeng [27] discovered the fiber whipping motion in slot-die melt blowing, appeared to be a fiber path with two groups of loops moving downward. These revealed fiber vibration or whipping motion indirectly confirms that air flow field in melt blowing is turbulent flow. However, few published papers on turbulent air flow field in melt blowing have been found, Xie and Zeng [28] preliminarily measured the fluctuating air velocity in slot-die melt blowing by hot-wire anemometer, it was regretful that the characteristic of temperature was not provided.

In the present work, our study still focused on discovering the characteristics of turbulent air flow field in slot-die melt blowing. Compared to the previous works [13-16, 28], innovations of researching turbulent air flow field in this work were: (1) an advanced two-dimensional velocity probe instead of one-dimensional velocity probe was applied in hotwire anemometer for measuring the fluctuation of air velocity, (2) the fluctuation of air temperature was originally measured, which has not been reported until now, (3) the coupling effect between air velocity and temperature was also analyzed by processing the data of measurements. 


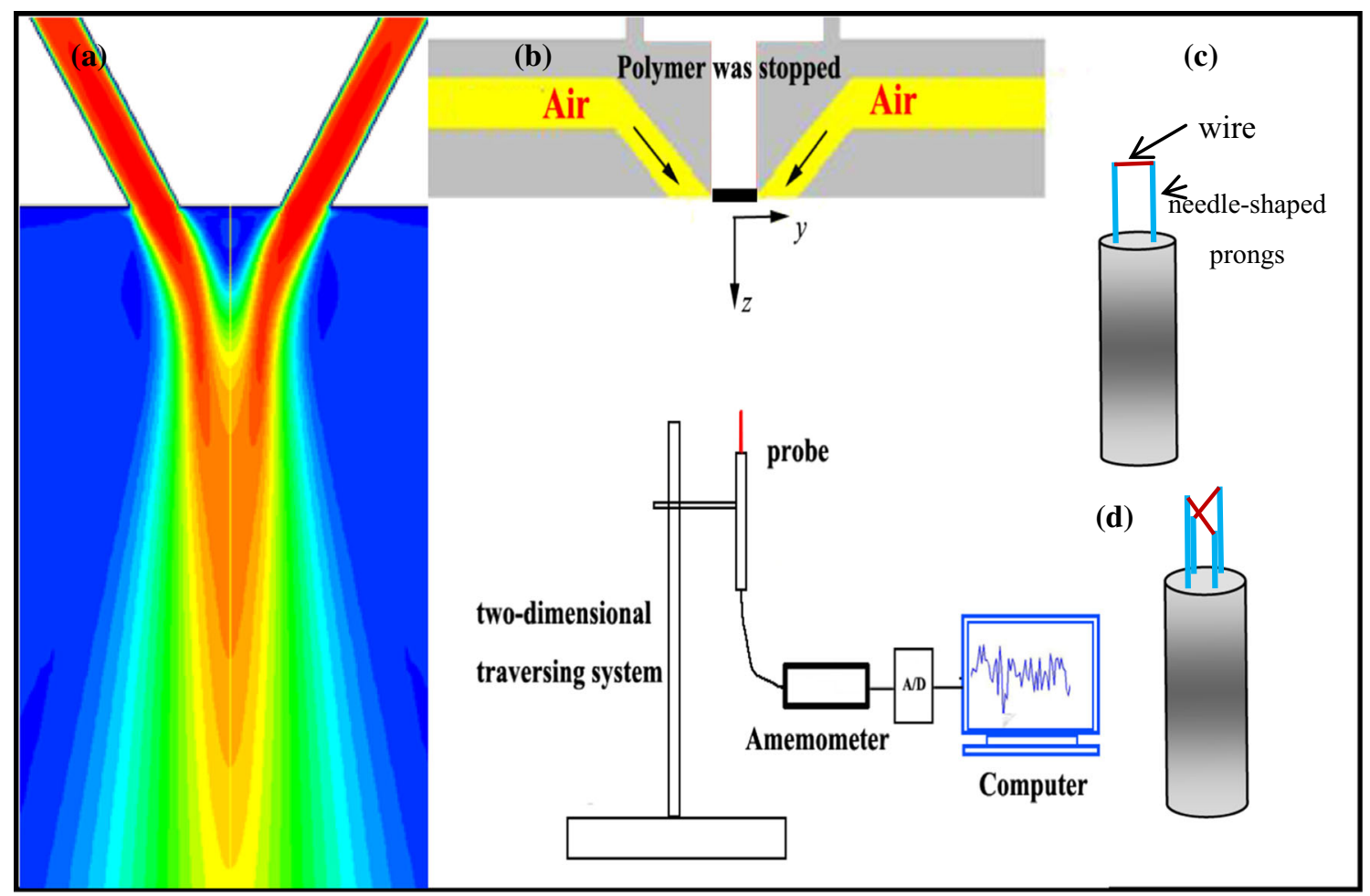

Figure 2 a The profile of air flow field in slot-die melt blowing which was obtained by CFD numerical simulation [29]. b Schematic of devices for measuring the air flow field in slotdie melt blowing. The anemometer probe was set below the die, c the structure of one-dimensional velocity probe used by previous

\section{Experiments and measurements}

\section{Melt-blowing setups and conditions}

The measurement of the air flow field in this study was taken on the single-orifice melt-blowing device; Fig. 1a shows the schematic of single-orifice melt blowing. The structure of this single-orifice slot die is shown in Fig. 1b, this type of die is referred to as a blunt-edge with a nose piece width $(f)$ of $1.28 \mathrm{~mm}$, a slot angle $(\alpha)$ of $30^{\circ}$, and a slot width $(e)$ of $0.65 \mathrm{~mm}$, the orifice diameter for polymer melt was $0.42 \mathrm{~mm}$. Figure 1c shows the real slot die used in this work. The details of this slot die can be found in Xie and Zeng [28]. The coordinate system used is also shown in Fig. 1a, all coordinates are relative to the die face. Its origin is at the center of the die face, the $x$ direction is along the major axis of the nose piece and slots, whereas the $y$ direction is transverse to the major axis of the nose piece and slots. The $z$ direction is directed vertically downward. researchers [14-16, 28], $\mathbf{d}$ the structure of two-dimensional probe used in the present work. It noted that the measurement of air flow field was taken in the absence of fiber. The air velocity and air temperature along $z$-axis were measured.

Our previous work [27] online measured the fiber whipping motion in slot-die melt blowing for the gauge air pressure of $1.0 \mathrm{~atm}$ (All air pressures used in this work refer to gauge pressures.), in view of discovering the relationship of turbulent air flow field and fiber motion, therefore, an air pressure of $1.0 \mathrm{~atm}$ was chosen again for measuring the turbulent air flow field. It is worth noting that, although the air pressure of $1.0 \mathrm{~atm}$ was higher than those used in previous work [23-26], it was still lower than the air pressure used in commercial manufacturing. For example, nonwoven fibers with diameter of $18 \mu \mathrm{m}$ could be produced with air pressure of $1.0 \mathrm{~atm}$ in this melt-blowing device, whereas the fiber diameter of the commercial nonwoven products was about 1-5 $\mu \mathrm{m}$. For air temperature, the mean air temperature can be measured by the thermocouples with high accuracy, in this study, we focused on the fluctuation of the air temperature; therefore, a low and a medium initial air temperatures of 50 and $100{ }^{\circ} \mathrm{C}$ were applied in consideration of the limitation of the measuring equipment (hot-wire anemometer). 


\section{Measurements}

Air velocity and temperature below the die were measured online with a hot-wire anemometer (Dantec StreamLine CTA90C10 and Dantec StreamLine CTA90C20, Dantec Dynamics, Skovlunde, Denmark) in the absence of the polymer stream, i.e., the polymer flow was stopped during measurements. Figure $2 \mathrm{~b}$ shows the relative positions of the hot-wire anemometer and the melt-blowing die.

During using hot-wire anemometer for measuring air velocity, the melt-blowing air takes away the heat of the wire, the temperature of the wire is kept constant by applying voltage on it (The constant temperature of the wire is kept $220^{\circ} \mathrm{C}$ higher than the ambient temperature, and the ambient temperature is $18{ }^{\circ} \mathrm{C}$.). The corresponding air velocity can be calculated from the applied voltage by the following equation

$\frac{E}{R_{\mathrm{w}}}=\left[\frac{\left(R_{\mathrm{w}}-R_{\mathrm{f}}\right)\left(A+B v^{1 / 2}\right)}{\alpha_{0} R_{\mathrm{f}} R_{\mathrm{w}}}\right]$

where $E$ is the heating voltage applied on the wire; $R_{\mathrm{w}}$ and $R_{\mathrm{f}}$ are the resistance of the wire at temperature of wire and air, respectively; $A$ and $B$ are constant numbers; $v$ is the air velocity and $\alpha_{0}$ is the temperature coefficient of resistance.

Compared to one-dimensional velocity probe used in previous researchers [14-16, 28], a two-dimensional velocity probe was attempted for the velocity measurement in this work; Figs. $2 \mathrm{c}$ and $2 \mathrm{~d}$ illustrate the structures of one-dimensional and two-dimensional velocity probe. The two-dimensional velocity probe is consisted of two systems of one-dimensional probes; each one-dimensional velocity probe has a metal wire with a 5- $\mu \mathrm{m}$ diameter and a 1.6-mm length suspended between two needle-shaped prongs. As shown in Fig. 2d, the two wires are placed in paralleled planes, the projection on $y-z$ plane and $x-$ $z$ plane of the two wires is vertical and paralleled, respectively. Compared to one-dimensional velocity probe for measuring air velocity, two-dimensional probe has better stability, for the reason that the wire undergoes drastic and continual air flow during measurement, the junction of wire and the needleshaped prongs or the wire itself probably become exfoliated or broken, resulting in incontinuity of measurement. However, if two-dimensional velocity probe is applied, the measurement will be suspended only in the case of the two wires are all disabled.
For the measurement of air temperature, a dedicated one-dimensional temperature probe (55P31) with a $1-\mu \mathrm{m}$ diameter and a 1.2-mm length metal wire was used. The structure of this one-dimensional temperature probe is similar to the one-dimensional velocity probe shown in Fig. 2c. This temperature probe operates at temperatures up to $150{ }^{\circ} \mathrm{C}$. Unlike the principle of velocity measurement, air temperature has linear relationship with applied voltage, namely

$T=C_{0}+C_{1} E$

where $T$ is the air temperature; $C_{0}$ and $C_{1}$ are coefficients and $E$ is the voltage provided from the hotwire anemometer.

During the measurements of air velocity and temperature, the corresponding velocity and temperature probe was positioned with a one-dimensional traversing system as shown in Fig. $2 b$, which permitted up or down motion along $z$-axis in 2-mm increments. It was found that during the measurements, although two-dimensional velocity probe was applied, the wires were easily broken when it was very close to the die; therefore, the minimum distance below the die was set to $z=6 \mathrm{~mm}$.

\section{Calibrations}

Calibrations including velocity calibration and temperature calibration were accomplished on calibration device at ambient temperature of $18^{\circ} \mathrm{C}$, before formal measurements.

For velocity calibration, the calibration device provided air with different rated velocities blowing to the wire, the corresponding voltages applied on the wire to maintain its temperature were also been collected, and the rated velocities and voltages were fitted into quartic-polynomial-equation, i.e., velocitycalibration curve. Later, during the formal measurement, the air velocities could be calculated from the voltage signals according to the known velocity-calibration curve. Because each wire in the two-dimensional velocity probe had an independent electrical circuit, two velocity-calibration curves were obtained simultaneously after each velocity calibration. Figure 3a shows the calibration curves of the two wires in two-dimensional velocity probe, and the two curves are almost coincided; however, the existed no coincided points illustrate that the microstructure of 
Figure 3 a The velocitycalibration curves for the two wires in the two-dimensional velocity probe. $\mathbf{b}$ The temperature-calibration curve for the one-dimensional temperature probe.

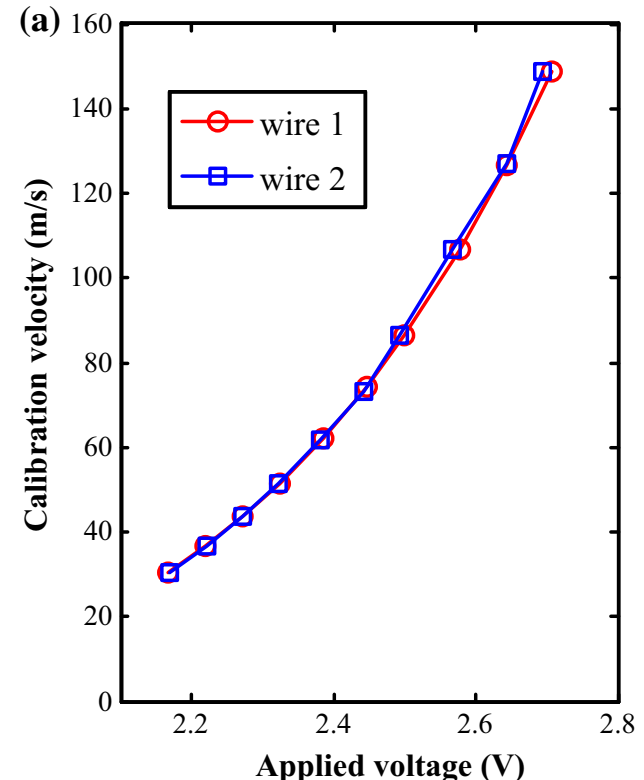

the two wires are not completely uniform. The quartic-polynomial-equation for wire 1 and wire 2 is

$v=0.00005 E^{4}+126.810379 E^{3}-691.024170 E^{2}$

$+1417.843384 E-870.765686$

$v=0.00007 E^{4}+152.901855 E^{3}-860.379089 E^{2}$

$+1687.597290 E-1141.561890$

For temperature calibration, the calibration air temperatures, $T$, were measured in advance by thermocouples, and the corresponding applied voltages, $E$, provided by hot-wire anemometer were also collected. Air temperatures, $T$, and the corresponding applied voltages, $E$, constitute the temperature-calibration curve, which is shown in Fig. $3 b$, and the linear equation for temperature calibration is

$T=3.9094 E-5.2452$

\section{Results and discussion}

\section{Fluctuation of velocity}

The instantaneous velocities at points along $z$-axis were measured by the hot-wire anemometer. The instantaneous velocity can be expressed as the sum of the mean and fluctuating velocities, namely $v_{t}=\bar{v}+v^{\prime}$. The instantaneous velocities, $v_{\mathrm{t}}$, at the points of $z=6,50$ and $100 \mathrm{~mm}$ as a function of time are shown in Fig. 4. A time segment of $0.2 \mathrm{~s}$ (from 0.5 to $0.7 \mathrm{~s})$ was chosen for demonstration of the instantaneous velocity. Figure 4 shows that the instantaneous velocity signal is irregular, and the fluctuating velocity, $v^{\prime}$, damps gradually as far away from the die (from $z=6 \mathrm{~mm}$ to $z=100 \mathrm{~mm}$ ). Figure 5 illustrates the probability distribution of the instantaneous velocity in twenty velocity sections; information of the maximum and minimum velocities as well as standard deviation of instantaneous velocity are also included. Probability distribution presents unimodal distribution. Here, we build two parameters, $\left|v^{\prime}\right|$ and $\Delta v$, for representing the turbulent characteristics of velocity. $\left|v^{\prime}\right|$ is absolute value of fluctuating velocity, $v^{\prime}$, which can represent the average intensity of the turbulence, $\Delta v$ is the range of the instantaneous velocity, $v_{t}$, which represents the amplitude changing of the instantaneous velocity. $\left|v^{\prime}\right|$ and $\Delta v$ are described as

$\left|v^{\prime}\right|=\frac{\sum_{1}^{N}\left|v_{t}-\bar{v}\right|}{N}$

where $v_{\mathrm{t}}$ and $\bar{v}$ are the instantaneous and mean fluctuating velocities; $N$ is the number of acquainted velocities in the time segment of $0.2 \mathrm{~s}$.

$\Delta v=\max \left(v^{\prime}\right)-\min \left(v^{\prime}\right)$

Figure 6a shows the detailed evolution of the fluctuating velocity, $\left|v^{\prime}\right|$, along $z$-axis. The maximum peak is at the point of $z=8 \mathrm{~mm}$, where as locating below the position where the two air streams merged [17]. This reveals that the fluctuation of the air flow 
Figure 4 The instantaneous velocity, $v_{\mathrm{t}}$, at the points a $z=6 \mathrm{~mm}$, b $50 \mathrm{~mm}$ and c $100 \mathrm{~mm}$ as a function of time, $t$.
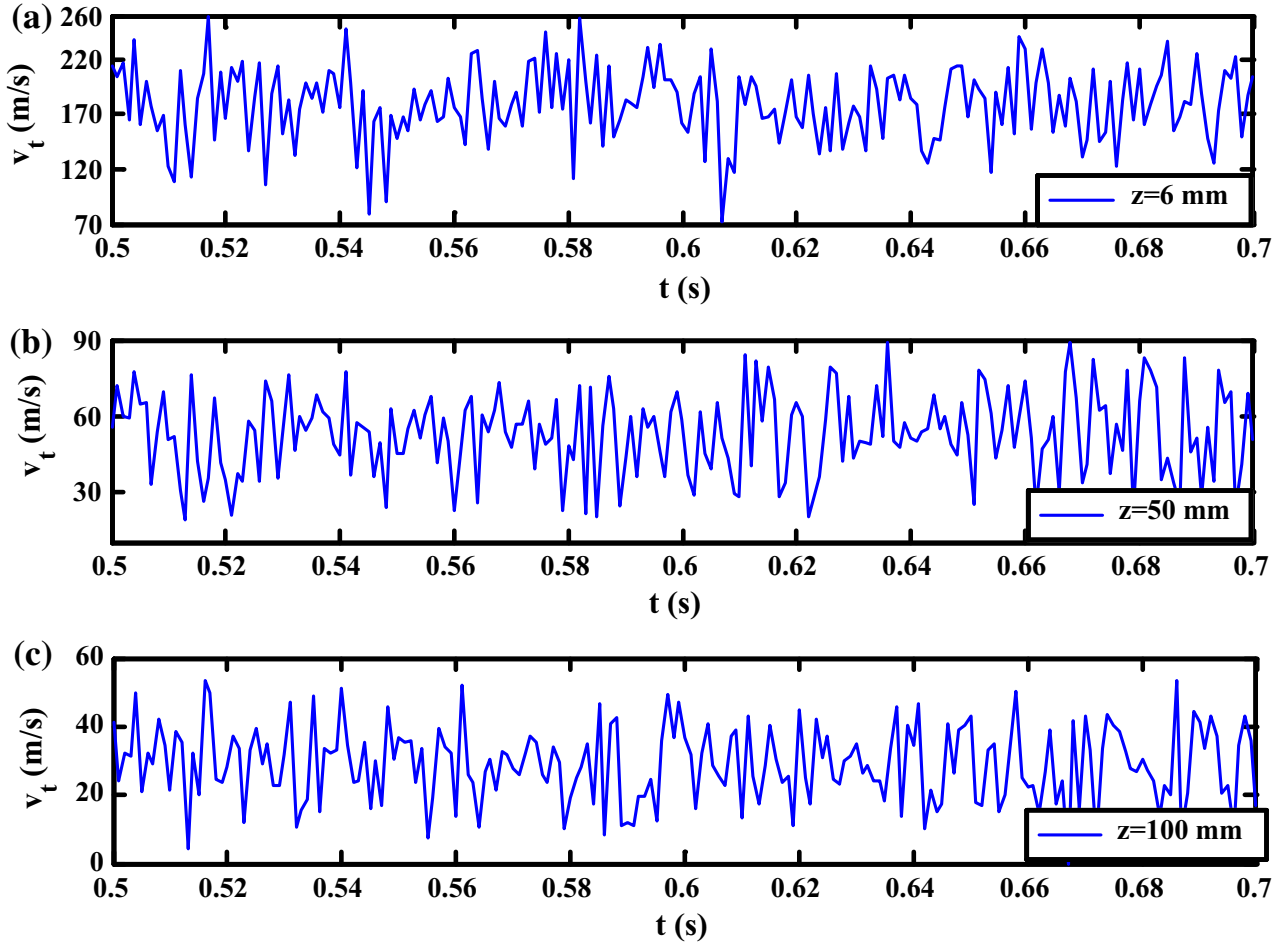
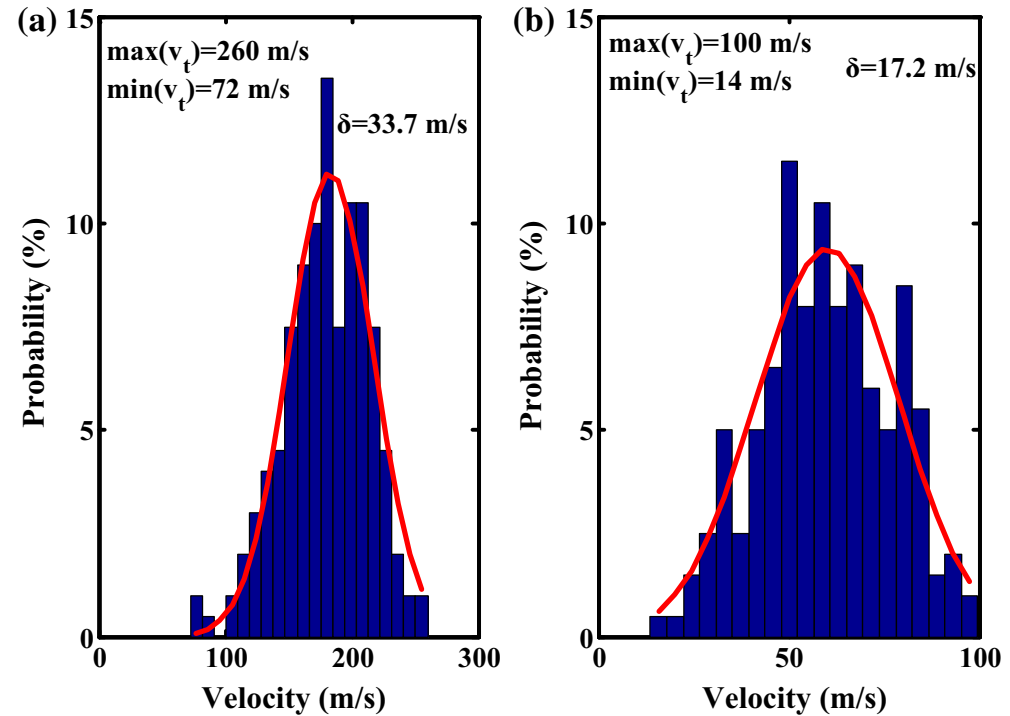

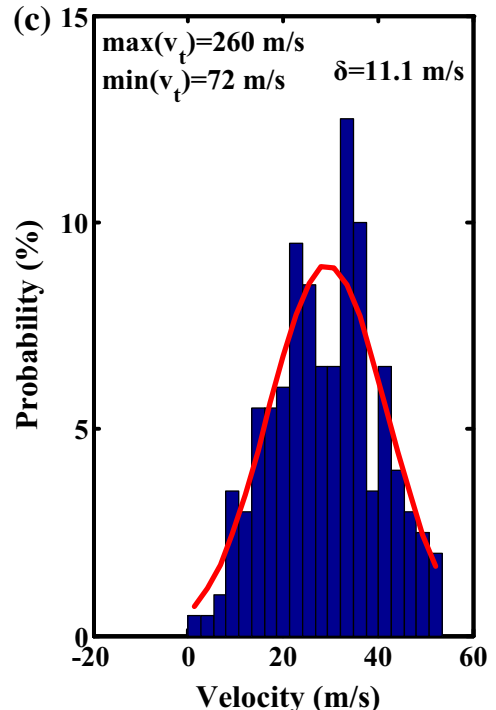

Figure 5 The probability distribution of the instantaneous velocity in twenty velocity sections for the positions of a $z=6 \mathrm{~mm}$, b $z=50 \mathrm{~mm}$ and c $z=100 \mathrm{~mm}$. These statistical results were

field continues increasing in the region where as several millimeters lag behind the merging point of the two air streams. It noted that an obvious second peak is located at the point of $z=14 \mathrm{~mm}$, although the reason for the second peak cannot be explicated according to the present knowledge we have, we believe the second peak hides some special obtained from Fig. 4. The $\delta$ is standard deviation of instantaneous velocity.

characteristics of turbulence in slot-die melt blowing. Figure $5 \mathrm{~b}$ shows the evolution of $\Delta v$ along $z$-axis, the velocity fluctuation is strong, for example, the maximum $\Delta v=185 \mathrm{~m} \mathrm{~s}^{-1}$ is at the point of $z=6 \mathrm{~mm}$, and even if at the point of $z=100 \mathrm{~mm}$, the $\Delta v$ is still as large as $50 \mathrm{~m} \mathrm{~s}^{-1}$. The obvious large $\Delta v$ plays a crucial role in inducing to the fluctuating attenuation 
Figure 6 The evolutions of a the fluctuating velocity, $\left|\mathrm{v}^{\prime}\right|$, and $\mathbf{b}$ the range of instantaneous velocity, $\Delta v$, along $z$-axis.

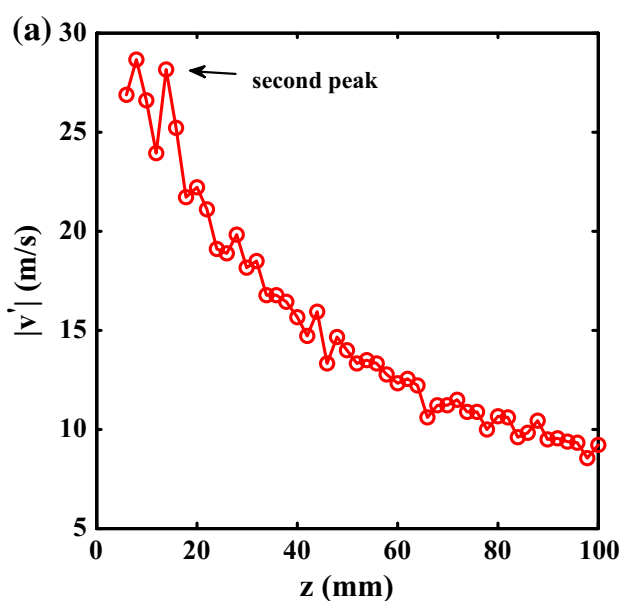

of the fiber in melt blowing, resulting in deteriorating the evenness of the nonwoven fibers. In addition, there exists a second peak of $\Delta v$ at the point of $z=14 \mathrm{~mm}$ which is interesting coincided with the point of the second peak of $\left|v^{\prime}\right|$ shown in Fig. 6a, we predict that, there may exist a steady vortex at the position near the point of $z=14 \mathrm{~mm}$. The fiber whipping motion in slot-die melt blowing discovered by our previous work [27] via high-speed photography appeared to be a fiber path with two groups of loops moving downward (see Fig. 7), the generation of the left-right alternating loops was accomplished coincidently in the region near the point of $z=14 \mathrm{~mm}$. It illustrates that the characteristic of turbulent air flow field has obvious effect on fiber formation during melt-blowing process.
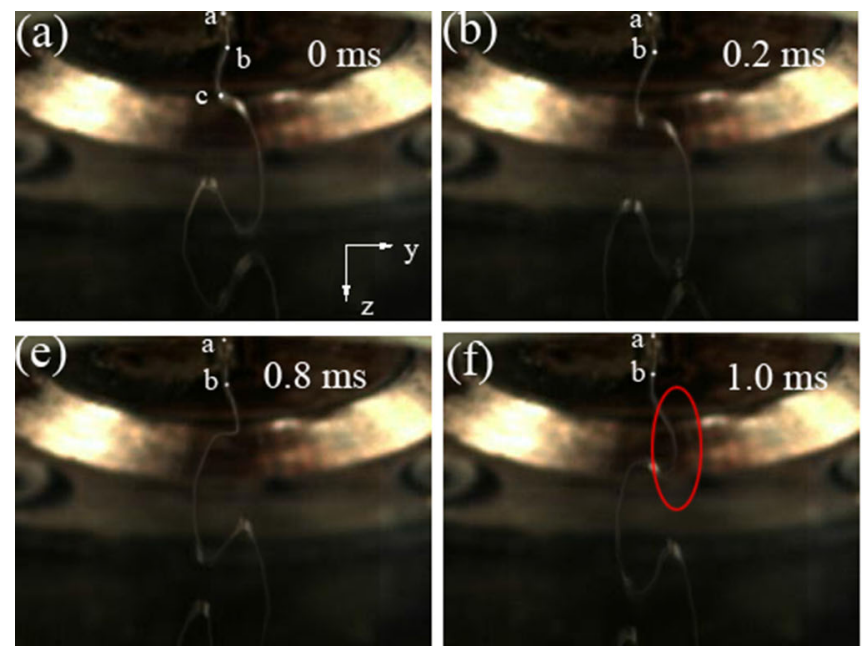

Figure 7 Evolutions of fiber paths below the slot die under experimental air pressure of $1.0 \mathrm{~atm}$ [27]. The real image size for all the images is $26.2 \mathrm{~mm} \times 19.6 \mathrm{~mm}$. The evolution of the left-

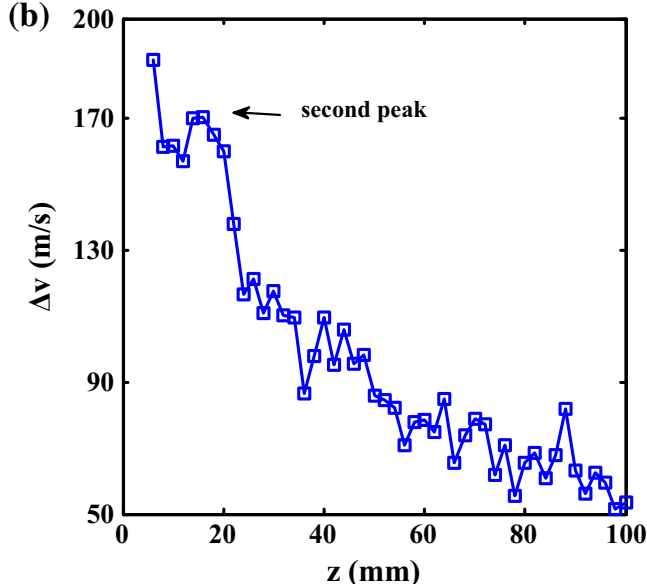

\section{Fluctuation of temperature}

Figure 8 shows the instantaneous air temperatures, $T_{\mathrm{t}}$, at the points of $z=6,50$ and $100 \mathrm{~mm}$, as a function of time, $t$. Figure 8 shows that the instantaneous temperature signal in time segment of 0.2 is as regular as sine or cosine curve. It is worth noting that there are ten peaks or troughs of signal-wave existed in time segment of $0.2 \mathrm{~s}$ at $z=6,50$ and $100 \mathrm{~mm}$, illustrates that the frequency of the sine or cosine curve-like instantaneous temperature signal is changeless no matter where the measuring points are. Correspondingly, Fig. 9 illustrates the probability distribution of the instantaneous temperature in twenty temperature sections. Comparing to probability distribution of instantaneous velocity shown in Fig. 5, the obvious difference is that the probability
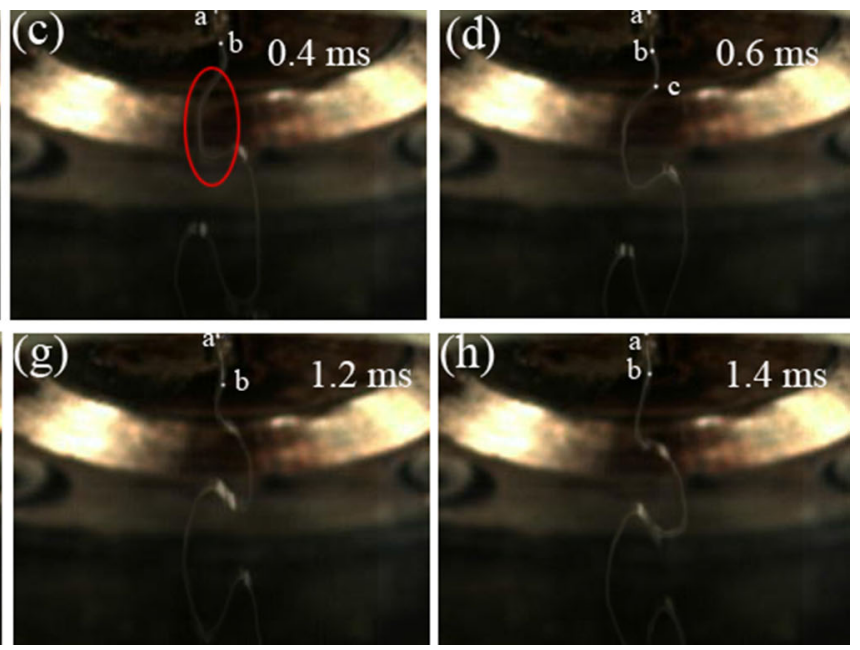

right alternating loops is also shown; fiber paths in the two elliptical annotations are the generated left and right loops. 
Figure 8 The instantaneous temperature, $T_{\mathrm{t}}$, at the positions of a $z=6 \mathrm{~mm}$, b $50 \mathrm{~mm}$ and c $100 \mathrm{~mm}$ as a function of time, $t$.
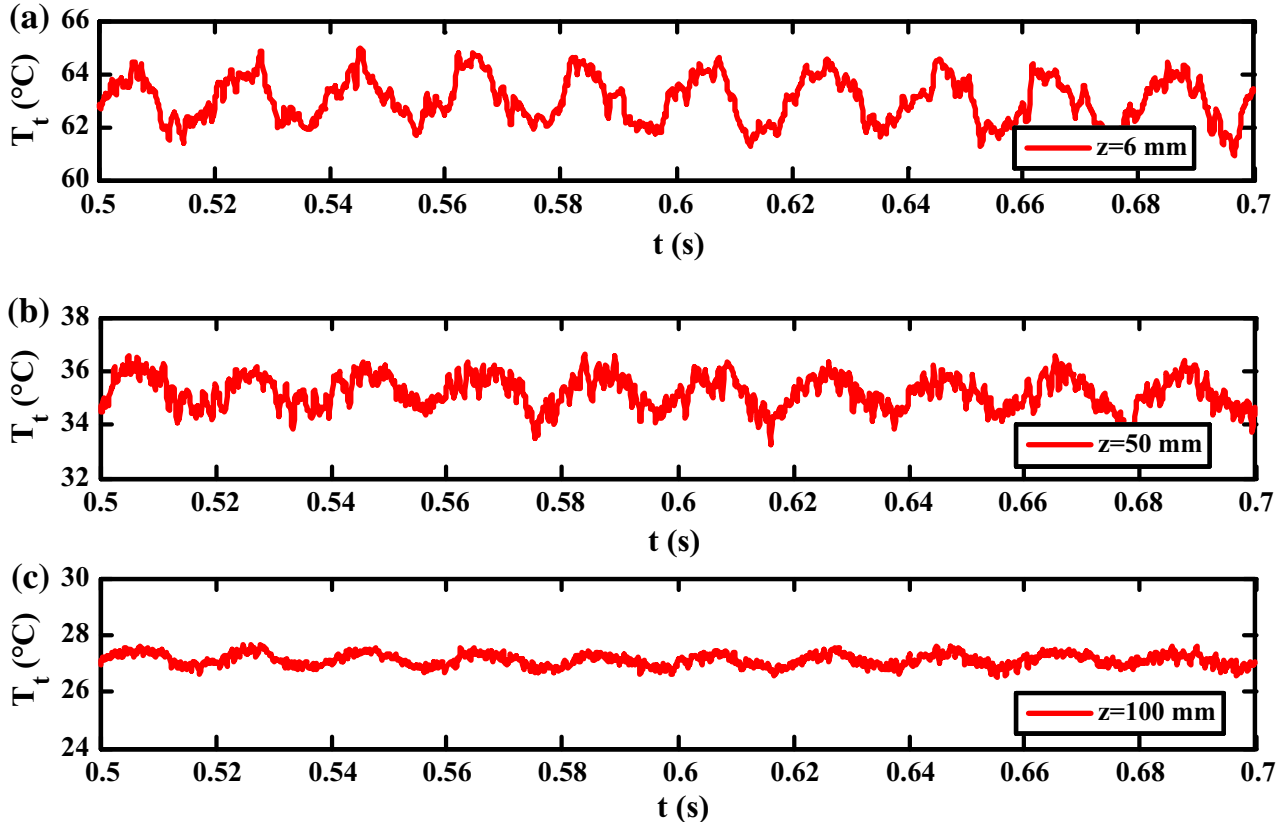

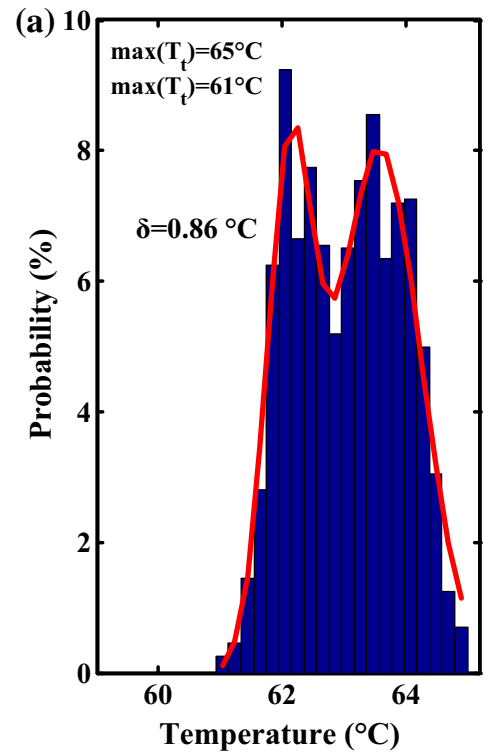

Figure 9 The probability distribution of the instantaneous temperature in twenty velocity sections for the positions of a $z=6 \mathrm{~mm}, \mathbf{b} z=50 \mathrm{~mm}$ and $\mathbf{c} z=100 \mathrm{~mm}$. These statistical

distribution of temperature presents bimodal distribution.

Similar to the definition of the instantaneous velocity, $v_{\mathrm{t}}$, the instantaneous air temperature, $T_{\mathrm{t}}$, is expressed as the sum of the mean and fluctuating temperatures, namely $T_{t}=\bar{T}+T^{\prime}$. A time segment of $0.2 \mathrm{~s}$ (from 0.5 to $0.7 \mathrm{~s}$ ) was chosen again for comparing the profile of temperature, $T_{t}$, with the

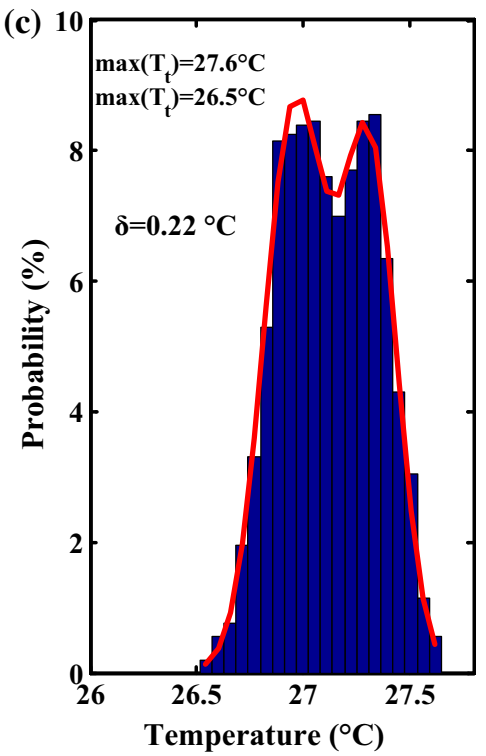

results were obtained from Fig. 8. The $\delta$ is standard deviation of instantaneous temperature.

instantaneous velocity, $v_{\mathrm{t}}$. Figure $10 \mathrm{a}$ and $\mathrm{b}$ shows the detailed evolution of the fluctuating temperature, $\left|T^{\prime}\right|$, and $\Delta T$, along $z$-axis. The parameters of $\left|T^{\prime}\right|$ and $\Delta T$ are defined similarly to $\left|v^{\prime}\right|$ and $\Delta v$ of the air velocity, as 
Figure 10 The evolutions of a the fluctuating temperature, $\left|T^{\prime}\right|$, and $\mathbf{b}$ the range of instantaneous temperature, $\Delta T$, along $z$-axis.

$\left|T^{\prime}\right|=\frac{\sum_{1}^{N}\left|T_{\mathrm{t}}-\bar{T}\right|}{N}$

where $T_{\mathrm{t}}$ and $\bar{T}$ are the instantaneous and mean fluctuating temperatures; $N$ is the number of acquainted temperatures in the time segment of $0.2 \mathrm{~s}$.

$\Delta T=\max \left(T^{\prime}\right)-\min \left(T^{\prime}\right)$

The maximum value of $\left|T^{\prime}\right|$ is about $0.82{ }^{\circ} \mathrm{C}$ as locating at the point of $z=6 \mathrm{~mm}$, and then decreased as far away from the die. Comparing to the $\left|v^{\prime}\right|$ shown in Fig. 6a, the obvious difference is that, there is no second peak existed, we deduce that the temperature fluctuation has little relationship with the velocity fluctuation, for the velocity is a vector, whereas the temperature is a scalar. As shown in Fig. $10 \mathrm{~b}$, the maximum value of $\Delta T=5.5^{\circ} \mathrm{C}$ is still at the point of $z=6 \mathrm{~mm}$ and then sharply decreases to about $4{ }^{\circ} \mathrm{C}$ at the point of $z=14 \mathrm{~mm}$, the $\Delta T$ continues decreasing gradually but with gradually increasing fluctuation in the region of $14 \mathrm{~mm} \leq z \leq 100 \mathrm{~mm}$. At the point of $z=6 \mathrm{~mm}$, $\Delta T=5.5^{\circ} \mathrm{C}$ is nonnegligible when comparing with the mean air temperature of $63{ }^{\circ} \mathrm{C}$ (shown in Fig. 11b in the next part). It is well known that temperature largely determines the molten fibers viscosity, and correspondingly affects the dynamic viscoelastic force of fiber. The obviously fluctuating $\Delta T$ in the region of $14 \mathrm{~mm} \leq z \leq 100 \mathrm{~mm}$ also contributes the fluctuating attenuation of dynamic viscoelastic force of fiber in melt blowing.

From Figs. 4 and 8, although air velocity and temperature have obviously different fluctuating characteristics in melt blowing, their characteristics of fluctuation play an important effect in fluctuating attenuation of the fiber. We suppose that the later research on fiber attenuation, fiber motion as well as the evenness of the nonwoven fibers in melt blowing should take the characteristics of fluctuating air velocity and temperature simultaneously into consideration.

\section{Mean velocity and temperature profiles}

Figure 11a shows the development of the mean air velocity profile as a function of the distance, $z$, along $z$-axis. It is shown that mean velocity decreases rapidly with increasing distance from the die until reaching the point of $z=50 \mathrm{~mm}$. A maximum mean velocity of $183 \mathrm{~m} \mathrm{~s}^{-1}$ is obtained at the point of $z=6 \mathrm{~mm}$, which is about $30 \mathrm{~m} \mathrm{~s}^{-1}$ higher than the maximum velocity measured by previous researchers [13-16]. Although air velocity in the region of $0 \mathrm{~mm}<z<6 \mathrm{~mm}$ is not shown in this work, it is no doubt that fiber undergoes strong attenuation effect by the high-speed air velocity in this region. However, as shown in Fig. 11a, the fiber velocity not increases rapidly but increases linearly and gradually, the measurement of the fiber velocity was described in our previous work [27], and the fiber was produced under the same air pressure using in this work. Moreover, in consideration of the fiber whipping paths shown in Fig. 7, we confirm that the turbulent air near the die with high-speed velocity results in inducing the lateral two-dimensional or three-dimensional whipping motion of the fiber, 
Figure 11 a The profile of measured mean air velocity along z-axis by hot-wire anemometer. The evolution of fiber velocity along $z$-axis [27] is added for comparison with air velocity. $\mathbf{b}$ The evolution profile of measured mean air temperature along $z$-axis, the initial temperature at $z=0 \mathrm{~mm}$ is $100{ }^{\circ} \mathrm{C}$.

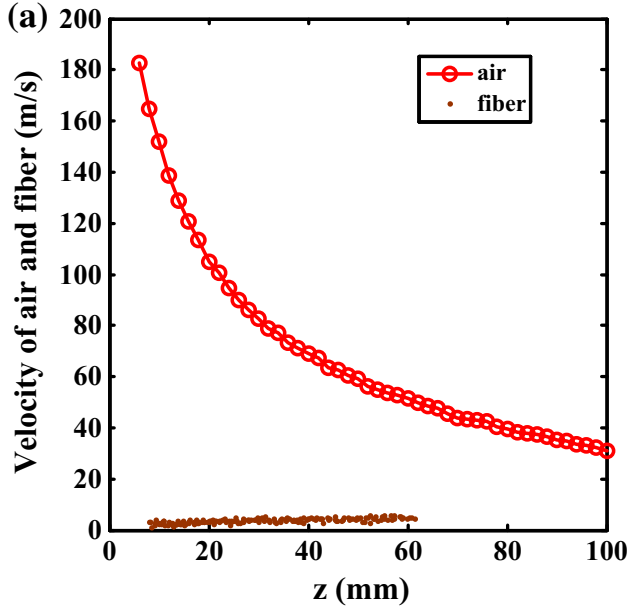

rather than rapidly increasing the fiber velocity along $z$ direction.

Figure $11 \mathrm{~b}$ shows the mean air temperature profiles along $z$-axis. The trend of the temperature decay is similar to the velocity decay shown in Fig. 11a. In view of the initial air temperature is $100{ }^{\circ} \mathrm{C}$ (air temperature at point of $z=0$ is $100{ }^{\circ} \mathrm{C}$ ), the air temperature decreases to be $50 \%$ of itself at the point of $z=13 \mathrm{~mm}$. It is this temperature drop that is believed to be the driving force for crystallization of the polymer melt and fiber solidification in the meltblowing process.

During melt-blowing process, both air velocity and temperature determine the attenuation of fiber, a lot of work have been done on optimizing the air flow field by designing new structure of the die [30, 31], these optimizations of air flow field were refer to that the new designed dies could provided air flow field with higher velocity and higher temperature, simultaneously. It is accessible that air with higher velocity can provide stronger attenuation of fiber and air with higher temperature can maintain molten status of fiber in longer time. However, Xie and Zeng [32] showed that higher air velocity and higher air temperature not always produced fibers with smaller diameter. In their work, a gauge air pressure of $1.0 \mathrm{~atm}$ created initial air velocities about 300 and $150 \mathrm{~m} \mathrm{~s}^{-1}$ for slot-die and swirl-die melt blowing; however, the fibers collected at $25-\mathrm{mm}$ distance below the die have a mean diameter of $75.2 \mu \mathrm{m}$ for the slot die, and $58.3 \mu \mathrm{m}$ for the swirl die, for the reason that the type of turbulence also determines attenuating ratio of fiber. In addition, we think that the energy wasting should be considered in optimizing air flow field in melt blowing, in other

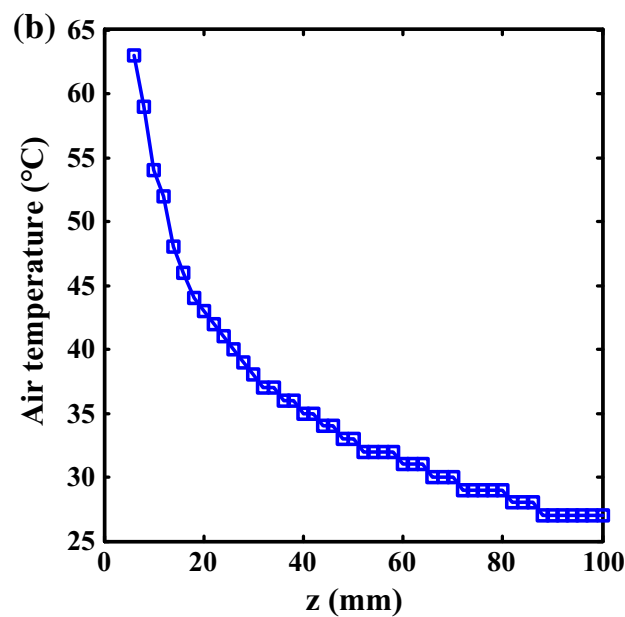

description, the attenuation effect provided by the relative velocity of air and fiber occurs just in the region where the fiber is at the status of molten; otherwise, high air velocity has no contribution to fiber attenuation where fiber is solidified, i.e., velocity energy wasting appears. Similarly, temperature wasting appears where fiber attenuation effect disappears, not only this, the molten fiber tends to rebound under its internal viscoelastic force resulting in fiber diameter re-increasing. The phenomenon of fiber rebound has been discovered [33, 34], which is very harmful to melt-blowing productions.

\section{Coupling effect of velocity and temperature}

In melt blowing, air velocity and temperature exist simultaneously for fiber attenuation. The coupling effect between air flow field and the fiber is the essential mechanical mechanism for fiber attenuation. Besides this coupling effect, the coupling effect of velocity and temperature may exist. In this part, we have done a tentative research on this coupling effect, in particular, the effect of temperature on velocity was revealed by processing data of measurements.

As mentioned above, before formal velocity measurement, the velocity calibration was carried out on calibrating device at ambient temperature, i.e., $18{ }^{\circ} \mathrm{C}$. Figure 12a shows the air velocities measured by hotwire anemometer at initial air temperature of 18, 50 and $100{ }^{\circ} \mathrm{C}$. For higher initial air temperature, the measured air velocity along $z$-axis is lower at any points below the die. Take the results at the point of $z=6 \mathrm{~mm}$ for example, the measured air velocities are about 182, 150 and $90 \mathrm{~m} \mathrm{~s}^{-1}$ at initial air temperature of 18,50 and $100{ }^{\circ} \mathrm{C}$. It noted that these 


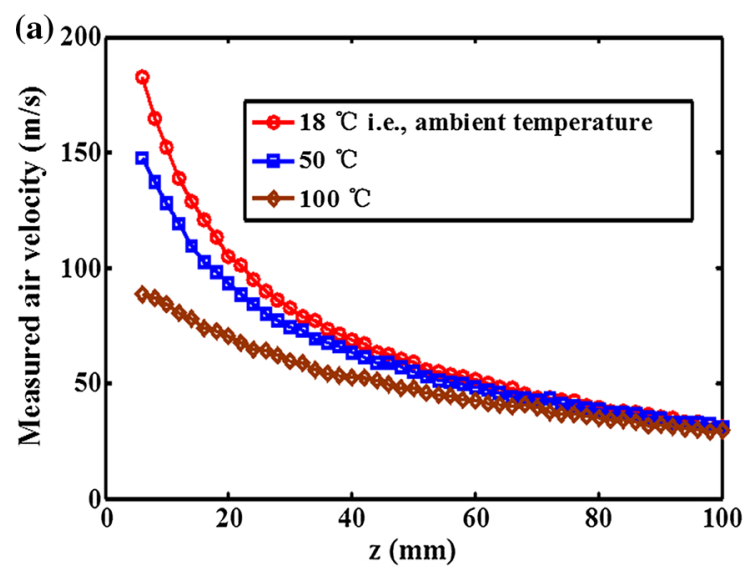

Figure 12 a The air velocities measured by hot-wire anemometer at initial air temperatures of 18,50 and $100{ }^{\circ} \mathrm{C}$. It noted that the measured air velocities by the hot-wire anemometer are calculated according to the velocity-calibration curves shown in Fig. 3a,

velocities are just measured values, rather than the real velocities. According to the principle of velocity measurement of hot-wire anemometer, melt-blowing air with higher temperature takes less temperature of the wire, resulting in lower voltage is needed for maintaining the constant temperature of the wire, the measured air velocity calculated by the velocity-calibration curve are corresponding lower. Fortunately, a correcting formula provided by hot-wire anemometer can be applied for converting the measured voltages applied on the wires at initial temperatures of 50 and $100{ }^{\circ} \mathrm{C}$, into the standard voltage applied on the wires at ambient temperature. As

$E_{\text {corr }}=\sqrt{\frac{T_{\mathrm{w}}-T_{0}}{T_{\mathrm{w}}-T}} \cdot E$

Here $E_{\text {corr }}$ is the converted voltage; $T_{\mathrm{w}}$ is the constant temperature of the wires, i.e., $238{ }^{\circ} \mathrm{C}$; $T_{0}$ is the ambient temperature, $18{ }^{\circ} \mathrm{C}$, and $T$ is the temperature of the melt-blowing air, $T$ at different points can be obtained from Fig. 9b.

By substituting $E_{\text {corr }}$ into the velocity-calibration curve shown in Fig. 3a, the corrected air velocities which are equal to the real velocities can be obtained. Figure $12 \mathrm{~b}$ shows the corrected air velocities as well as the standard air velocity measured at ambient temperature, along $z$-axis (The inset shows the air velocities on an expanded scale.). Although Fig. 12b illustrates that the profile of air velocities along $z$-axis are almost coincided with each other at different initial air temperatures, there still exists a nonnegligible difference of the air velocities by analyzing the

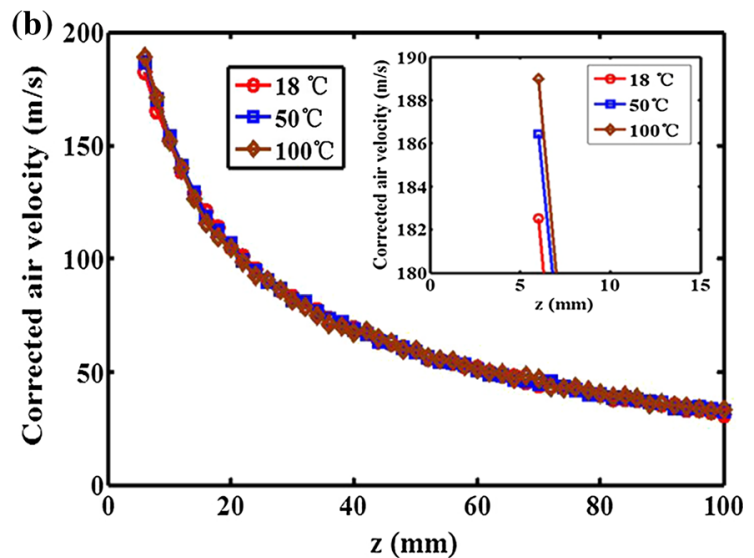

b the corrected air velocities as well as the standard air velocity measured at ambient temperature, along $z$-axis (the inset shows the air velocities on an expanded scale).

inset of Fig. 12b. The inset shows that temperature has an enhanced effect on velocity, at the point of $z=6 \mathrm{~mm}$, air velocities are about 182.5, 186 and $188.5 \mathrm{~m} \mathrm{~s}^{-1}$ at the initial air temperatures of 18,50 and $100{ }^{\circ} \mathrm{C}$, respectively. Actually, air with initial temperatures of 50 and $100{ }^{\circ} \mathrm{C}$ decrease to about 35 and $63{ }^{\circ} \mathrm{C}$, respectively, at $z=6 \mathrm{~mm}$. In other words, air temperature difference of $45{ }^{\circ} \mathrm{C}$ (i.e., $45=63-$ $18{ }^{\circ} \mathrm{C}$ ) generates velocity difference of $6 \mathrm{~m} / \mathrm{s}$ (i.e., $6=188.5-182.5 \mathrm{~m} \mathrm{~s}^{-1}$ ). In commercial melt blowing, the initial air temperature is commonly set to about $300{ }^{\circ} \mathrm{C}$ or above, moreover, with in view of Fig. 10b, we speculate that the temperature fluctuation, $\Delta T$, will become larger in commercial condition, correspondingly resulting in enhanced fluctuation of the air velocity. Fluctuations of both velocity and temperature may account for the reason why the evenness of fiber diameter produced by melt blowing is poor. The typical melt-blowing fibers with poor diameter evenness are shown in Fig. 13a. Figure 13a shows the SEM image of fibers produced by the meltblowing equipment described in this work, using a scanning electron microscope (JSM-5600LV, JEOL, Tokyo, Japan), Fig. 13b illustrates the corresponding probability distribution of the fiber diameters.

\section{Conclusions}

Turbulent air flow field in slot-die melt blowing was experimentally studied by using hot-wire anemometer. The fluctuating characteristics of the 
Figure 13 a The SEM image of fibers produced by the meltblowing equipment described in this work, using a scanning electron microscope (JSM5600LV, JEOL, Tokyo, Japan), b the corresponding probability distribution of the fiber diameters.
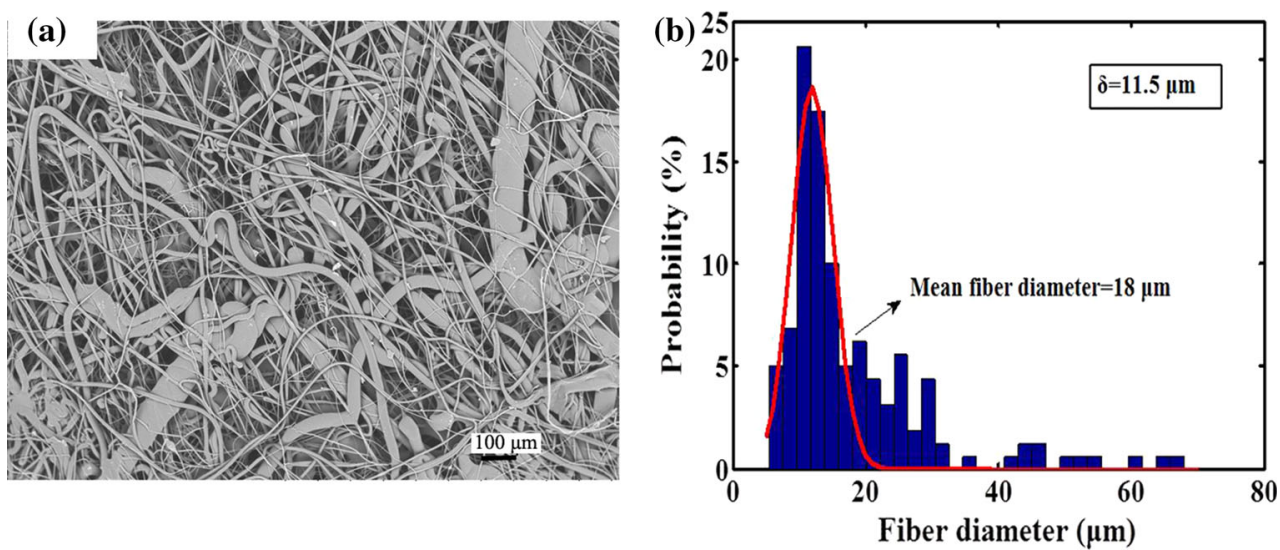

velocity and temperature as well as mean velocity and temperature were obtained. The results showed that the characteristics of velocity and temperature fluctuations were obviously different, the velocity fluctuation was irregular and strong, and it contained some unexplored characteristics of the turbulence, such as a predicted steady vortex existed at the position near $z=14 \mathrm{~mm}$. The fluctuation of temperature revealed a regular profile like sine or cosine curve, moreover, the frequency of the sine or cosine curve was changeless at different points along $z$-axis. In the last part of this study, the coupling effect of velocity and temperature was analyzed; the results showed that temperature has an enhanced effect on velocity. The fluctuation of temperature not only contributed to changing of viscosity of the fiber but also generated fluctuation of velocity. Fluctuation of both velocity and temperature could account for the reason why the evenness of fiber diameter produced by melt blowing was poor.

This work discovered that the characteristics of turbulent air flow field had great relationship with the fiber motion as well as fiber diameter evenness of melt-blowing products. Indicated that turbulent air flow field deserved to be further discovering in order to fully research on melt blowing.

\section{Acknowledgements}

This research was supported by the National Natural Science Foundation of China (Grant Nos. 11702113 and 51506075), Natural Science Foundation of Zhejiang Province (Grant Nos. LQ18E040001 and LQ18E030013) and Educational Commission of Zhejiang Province of China (Y201636479).

\section{Compliance with ethical standards}

Conflicts of interest We declare that we have no conflict of interest.

Open Access This article is distributed under the terms of the Creative Commons Attribution 4.0 International License (http://creativecommons.org/ licenses/by/4.0/), which permits unrestricted use, distribution, and reproduction in any medium, provided you give appropriate credit to the original author(s) and the source, provide a link to the Creative Commons license, and indicate if changes were made.

\section{References}

[1] Zhang Z, Chwee TL, Ramakrishna S, Huang ZM (2005) Recent development of polymer nanofibers for biomedical and biotechnological applications. J Mater Sci Mater Med 16:933-946. https://doi.org/10.1007/s10856-005-4428-x

[2] Burger C, Hsiao BS, Chu B (2006) Nanofibrous materials and their applications. Annu Rev Mater Res 36:333-368

[3] Krutka HM, Shambaugh RL, Papavassiliou DV (2002) Analysis of a melt-blowing die: comparison of CFD and experiments. Ind Eng Chem Res 41:5125-5138

[4] Krutka HM, Shambaugh RL, Papavassiliou DV (2003) Effects of die geometry on the flow field of the melt-blowing process. Ind Eng Chem Res 42:5541-5553

[5] Krutka HM, Shambaugh RL, Papavassiliou DV (2004) Effects of temperature and geometry on the flow field of the melt blowing process. Ind Eng Chem Res 43:4199-4210

[6] Krutka HM, Shambaugh RL, Papavassiliou DV (2005) Analysis of multiple jets in the schwarz melt-blowing die using computational fluid dynamics. Ind Eng Chem Res 44:8922-8932 
[7] Krutka HM, Shambaugh RL, Papavassiliou DV (2006) Analysis of the temperature field from multiple jets in the schwarz melt blowing die using computational fluid dynamics. Ind Eng Chem Res 45:5098-5109

[8] Harpham AS, Shambaugh RL (1996) Flow field of practical dual rectangular jets. Ind Eng Chem Res 35:3776-3781

[9] Harpham AS, Shambaugh RL (1997) Velocity and temperature fields of dual rectangular jets. Ind Eng Chem Res 36:3937-3943

[10] Tate BD, Shambaugh RL (1998) Modified dual rectangular jets for fiber production. Ind Eng Chem Res 37:3772-3779

[11] Tate BD, Shambaugh RL (2004) Temperature fields below melt-blowing dies of various geometries. Ind Eng Chem Res 43:5405-5410

[12] Rovere A, Shambaugh RL (2001) Melt-spun hollow fibers for use in nonwoven structures. Ind Eng Chem Res 40:176-187

[13] Lee YE, Wadsworth LC (2007) Fiber and web formation of melt-blown thermoplastic polyurethane polymers. J Appl Polym Sci 105:3723-3727

[14] Chen T (2003) Study on the air drawing in melt blowing nonwoven process. PhD Dissertation, Donghua University

[15] Sun YF, Liu BW, Wang XH, Zeng YC (2011) Air-flow field of the melt-blowing slot die via numerical simulation and multiobjective genetic algorithms. J Appl Polym Sci 122:3520-3527

[16] Wang YD, Wang XH (2014) Experimental investigation into a new melt-blowing die for dual rectangular jets. Adv Mater Res 985-949:270-273

[17] Moore EM, Shambaugh RL, Papavassiliou DV (2004) Analysis of isothermal annular jets: comparison of computational fluid dynamics and experimental data. J Appl Polym Sci 94:909-922

[18] Ellison CJ, Phatak A, Giles DW, Macosko CW, Bates FS (2007) Melt blown nanofibers: fiber diameter distributions and onset of fiber breakup. Polymer 48:3306-3316

[19] Tan DH, Zhou CF, Ellison CJ, Kumar S, Macosko CW, Bates FS (2010) Meltblown fibers: Influence of viscosity and elasticity on diameter distribution. J Non-Newtonian Fluid Mech 165:892-900

[20] Zhou CF, Tan DH, Janakiraman AP, Kumar S (2011) Modeling the melt blowing of viscoelastic materials. Chem Eng Sci 66:4172-4183
[21] Chung C, Kumar S (2013) Onset of whipping in the melt blowing process. J Non-Newtonian Fluid Mech 192:37-47

[22] Tan DH, Herman PK, Janakiraman A, Bates FS, Kumar S, Macosko CW (2012) Influence of Laval nozzles on the air flow field in melt blowing apparatus. Chem Eng Sci $80: 342-348$

[23] Chhabra RC, Shambaugh RL (1996) Experimental measurements of fiber threadline vibrations in the melt-blowing process. Ind Eng Chem Res 35:4366-4374

[24] Bresee RR (2005) Influence of processing conditions on melt blown web structure: part 2-primary airflow rate. Int Nonwovens J 14:11-18

[25] Bresee RR (2005) Influence of processing conditions on melt blown web structure: part III-water quench. Int Nonwovens J 14:27-35

[26] Bresee RR, Qureshi UA (2006) influence of processing conditions on melt blown web structure: part IV-fiber diameter. Int Nonwovens J 1:32-46

[27] Xie S, Zeng YC (2013) Online measurement of fiber whipping in the melt-blowing process. Ind Eng Chem Res 52:2116-2122

[28] Xie S, Zeng YC (2013) Turbulent air flow field and fiber whipping motion in the melt blowing process: experimental study. Ind Eng Chem Res 51:5346-5352

[29] Sun YF, Zeng YC, Wang XH (2011) Three-dimensional model of whipping motion in the processing of macrofibers. Ind Eng Chem Res 50:1099-1109

[30] Sun YF, Wang XH (2011) Optimal geometry design of the melt-blowing slot die with high stagnation temperature via the orthogonal array method and numerical simulation. J TEXT I 102:65-69

[31] Wang YD, Zhang HD, Lu TY (2016) Improvement of airflow field in melt-blowing processing. Ind Textila 67:238-243

[32] Xie S, Zheng YS, Zeng YC (2014) influence of die geometry on fiber motion and fiber attenuation in the melt-blowing process. Ind Eng Chem Res 53:12866-12871

[33] Helian XW (2012) study on the processing parameters for producing meltblown microfibers. Master's Thesis, Donghua University

[34] Xie S, Han WL (2017) Simulation and verification of fiber diameter re-increasing in melt blowing process. J Text Res $38: 17-21$ 